\title{
Research and Implementation of a Boost Semiconductor Lighting Driver with Average Current Double Loop Control
}

\author{
Renbo $\mathrm{Xu}^{1,2}$,Shaoyi $\mathrm{Li}^{1}$ and Zongsheng $\mathrm{Nie}^{1}$
}

School of artificial intelligence, Nanchang Institute of Science and Technology, Nanchang 330108, china. 2.Physics and Microelectronics Institute, Central South University, Changsha 410083 china

Keywords: Average Current;Double Loop Control;Semiconductor Lighting; Efficiency and Stability

\begin{abstract}
Based on the analysis of the common control methods of Boost converters, a new type of control strategy for the drive is proposed. Auxiliary inductance snubber circuits and a new method of average current double loop control of function generators based on the traditional average current principle is adopted to establish new current control boost drive control system based on function generator. It eliminates the multiplier in the traditional control method and does not need to detect the inductor current. It has the advantages of simple implementation, strong anti-interference ability, fast response speed, and low input current harmonic components.
\end{abstract}

\section{Introduction}

With the widespread application of power electronic devices such as semiconductor lighting, the problem of current harmonics in the power grid is increasingly serious, and harmonic pollution brings a series of harm to the power itself and the surrounding electromagnetic environment. Efficient lighting-driven APFC technology has also become a new research focus in power electronics technology. At present, commonly used APFC technologies include average current, current hysteresis, and current peak control techniques. The traditional average current control, current hysteresis control, and current peak control techniques require the use of inductance signal detection loops and multipliers. This leads to complex control structures and high circuit costs, and the nonlinear distortion of the multipliers greatly increases the system current. Harmonic content. In this paper, Boost type is taken as an example to propose a new type of efficient current predictive average current control technology based on function generator. The fact is that the reset switch is driven by the RS flip-flop $\bar{Q}$ instead of the clock signal, which makes the integrator capacitor is reset only during power switched off, not during power switched on, and its integrator capacitor reset time does not affect the function waveform, which also reduces system cost, THD EMI, and switch stress.

\section{Analysis of Traditional Average Current Control Method}

The average current mode control (ACMC) method is currently used more often. By controlling the average value of the current, it can reach the same phase as the input rectified voltage to achieve power factor correction, and the output voltage is stable, the current loop has a higher gain bandwidth, the tracking error produces a small distortion, easy to approach the power factor of 1 . In addition, it is not sensitive to squeaking and has high stability, so it has been widely used. The circuit principle is shown in Fig. 1, the input current signal is directly detected by the inductance signal and then compared with the reference current signal. The high frequency component change is averaged by the current error amplifier, and the amplified current error signal is compared with the sawtooth signal, which will then give pulse width modulation (PWM) to the main switching signal Q, whose signal performance determines the duty cycle of the main switch to make the inductor current approaches its average value. For example, when the inductor current $\mathrm{I}_{\mathrm{L}}$ rises, the PWM comparator output duty cycle decreases, reducing the current. If the output voltage decreases, the output of the voltage error comparator signal will increase, making the reference signal output 
by the multiplier larger, the current increasing, and the output voltage rising. On the contrary, if the inductor current becomes smaller, the output voltage is reduced, the system voltage is stable and the power factor is high. However, when inductance signal detection loops and multipliers are needed, the control structure will be complex and the circuit cost is high. In addition, the nonlinear distortion of the multiplier greatly increases the harmonic content of the system current.

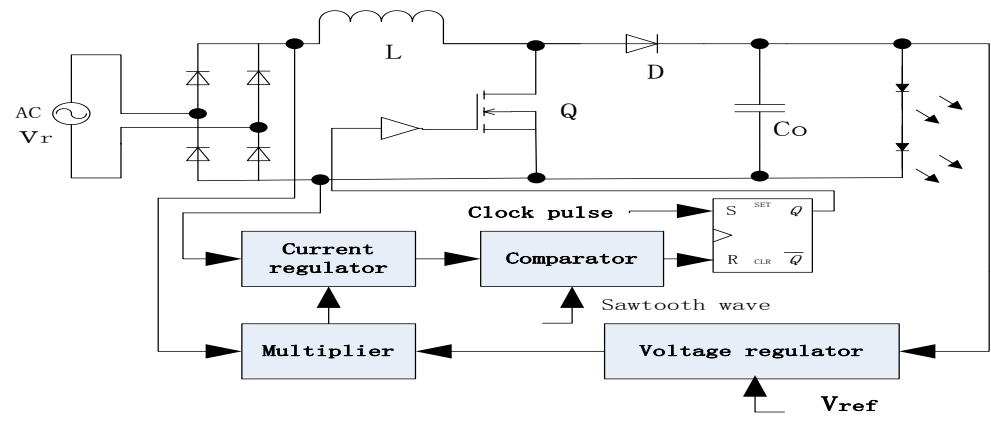

Figure 1. ACMC-based Boost LED Driver

\section{Basic Ideas and Strategies of Boost Average Current Control}

The new current control method of the proposed function generator is based on the system differential constraint relationship, which uses the known state quantity to make predictive control of the system current in one switching cycle to predict the current at the next sampling time, and then an optimal control strategy is used to calculate the current control state so that the current error is minimized and the actual current at the next sampling moment is forced to track its reference current with an optimal characteristic to achieve the corresponding function. The inductance signal detection loop and the multiplier are not used in the control circuit. The duty cycle in all the one half-waves will be generated by the predictive control strategy in the first half-wave, and then the current control strategy is determined according to the error between the two. Predictive current control technology is more in line with the actual characteristics of the controlled process. This control method eliminates the multiplier in the conventional average current control method, does not need to detect the inductor current, simplifies the control structure, avoids the harmonic content of the input current due to the nonlinear distortion of the conventional multiplier, and also reduces the system cost, has no low-frequency oscillation, and power factor is closer to 1, has system stability and greatly improves performance.

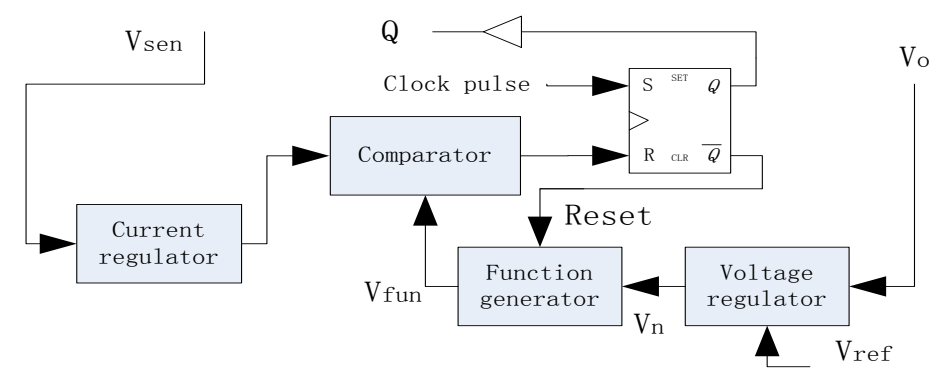

Figure 2. Structure Diagram of New Current Control Based on Function Generator

The system control principle is shown in Fig. 2. There is no inductance detection loop and traditional multiplier, and a function generator that generates optimal control is added. It can be seen from the figure that the output voltage $V_{O}$ of the LED drive system is fed back to the voltage regulator, and then a voltage error signal $V_{n}$ is generated after being compared with the reference voltage signal $V_{\text {ref }}$ and sent to a function generator to generate a function output signal $V_{\text {fun }}$ with an optimal control strategy, which is used as a current comparison reference, also known as the current tracking signal. The rectified input current $i_{i n}$ is sensed by the sense resistor $R_{\text {sen }}$ and fed back to 
the current regulator. After passing through the current regulator, it is compared with the current reference signal output by the function generator and sent to the reset terminal of the RS trigger. The output signal of the trigger $Q$ and clock signal work together to drive the power switch tube and the trigger terminal $\bar{Q}$ is used to reset the function generator, so that the error of this cycle does not affect the next switching cycle, and also generates the reference current value of the next sampling current. The drive control is performed by a function generator with an optimal control strategy, so that the input current $i_{i n}$ tracks the input voltage $v_{i n}$ well, the power factor of the entire Boost driver is greatly improved, and the output voltage $V_{O}$ remains stable.

The optimal control strategy of the function generator will be analyzed as follows. In this paper, only the continuous conduction mode is studied. The current control waveform of the new current control method based on function generator in one cycle is shown in Figure 3.

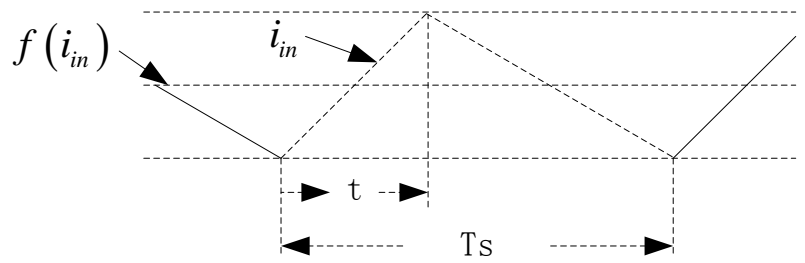

Figure 3 Current Control Waveform in One Cycle

The purpose of reducing the harmonic pollution and increasing the power factor is to make the input current follow the input voltage and the phase difference is zero, ie:

$$
f\left(i_{\text {in }}\right)=v_{\text {in }} / R_{\mathrm{e}}
$$

Among them, the $i_{i n}$ is rectifier input current, $v_{i n}$ is input voltage, $R_{\mathrm{e}}$ is the system equivalent resistance. The new current control method based on function generator works through the optimal control function generator, making the system main current zero phase track the rectified input voltage. It can be seen from Figure 3 that in order to make the average value of the inductor current $i_{\text {in }}$, which is the input current, equal to the average value of the current function $f\left(i_{i n}\right)$, the control strategy of the new current control method based on the function generator is:

$$
i_{i n, t}=\frac{v_{i n}}{R_{e}}+\frac{V_{O}-v_{i n}}{2 L}(1-D) T_{S}=f\left(i_{i n}\right)+\frac{\left(V_{O}-v_{i n}\right)\left(T_{S}-t\right)}{2 L}
$$

Based on the basic analysis of the main circuit of the Boost converter, combined with the parameters in Figure 3, the relationship between the input voltage and output voltage in the continuous conduction mode is:

$$
V_{o} / v_{\text {in }}=1-t / T_{S}
$$

In combination with 2,3 , there is a new current control Boost LED driver control strategy based on a function generator:

$$
i_{i n, t}=\frac{V_{O}}{R_{e}}\left(1-t / T_{S}\right)+\frac{V_{O}}{2 L}\left(t-t^{2} / T_{S}\right)
$$

Because $\frac{V_{O}}{R_{e}}=\frac{V_{n}}{R_{\text {sen }}}$ the control strategy for function generator includes: 


$$
\begin{aligned}
& V_{\text {fun }}(t)=i_{\text {in }, t} R_{\text {sen }}=V_{n}\left(1-t / T_{S}\right)+\frac{V_{O} R_{\text {sen }}}{2 L}\left(t-t^{2} / T_{S}\right) \\
& =-\frac{V_{O} R_{\text {sen }}}{2 L T_{S}} t^{2}+\left(\frac{V_{O} R_{\text {sen }}}{2 L}-\frac{V_{n}}{T_{S}}\right) t+V_{n}
\end{aligned}
$$

From the above analysis, it can be seen that a function generator is used to generate a reference signal for current comparison according to the above relative parameter relationship, and the input current will follow the input voltage well to achieve a high power factor, and the voltage output is stable and efficient.

\section{Conclusion}

This article has created a new current-controlled Boost drive system based on a function generator. Emphasis is placed on the design analysis of the auxiliary inductor current and function generator current control Boost drive system. The control system does not use the multiplier in the traditional control method and does not need to detect the inductor current. It has the advantages of simple implementation, strong anti-interference ability, fast response, low input current harmonic components, elimination of low-frequency oscillation, power factor close to 1 , wide input voltage and load variation range, which can meet the trend of simplification and high-efficiency stabilization of current power factor correction control structures.

\section{Acknowledgement}

Project Funding: Jiangxi Educational Science Planning Topics (No.17YB252); Nanchang DME Photoelectric Engineering Key Laboratory (No.NCZDSY-004); Nanchang Institute of Science \& Technology Research Fund (No. NGRCZX-17-01)

\section{References}

[1]Y. Hu, M. Jovanovic. LED Driver with Self-Adaptive Drive Voltage. IEEE Transactions on Power Electronics, 2008, 23(6): 116-125

[2]Y. K. Cheng, K. W. E. Cheng. General Study for Using LED to replace Traditional Lighting Devices. 2nd International Conference on Power Electronics Systems and Applications, Hong Kong, 2012, 25(9):173-177

[3] H. van der Broeck, G. Sauerlander, M. Wendt. Power Driver Topologies and Control Schemes for LEDs. IEEE Applied Power Electronics Conference, Anaheim, 2007, 31(5):319-325

[4]B. Wang, X. Ruan, K. Yao. A Method of Reducing the Peak-to-Average Ratio of LED Current for Electrolytic Capacitor-Less AC-DC Drivers. IEEE Transactions on Power Electronics, 2010, 25(3): 592-601

[5]Xiaoqun Wu, Chi K. Tse. Fast-Scale Instability of Single-Stage Power-Factor-Correction Power Supplies. IEEE, 2008, 37(5):477-480.

[6]J. P. Noon. Designing High-Power Factor Off-Line Power Supplies. Proceedings of Unitrode power Supply Design Seminar Manual SEM1500, Texas Instruments, 2007, 43(2):2-6.

[7] M Dyble, Narendran N, Bierman A. Impact of dimming white LEDs: chromaticity shifes due to different dimming methods. Proc. SPIE, 2008, 35(4):1 9

[8]K Hwu. and Chou S.. A simple current-balancing converter for LED lighting. IEEE Applied Power Electronics Conference, Washington DC, USA, 2009， 35(9):16-19 